\title{
LETTER / LETTRE
}

January 27, 2011

Sophie M. Regalado

Editor, JCHLA/JABSC

Ms. Regalado,

We would like to voice our concerns about the methodology presented in the article The Provision of Bioinformatics Services in Canadian Academic Libraries (JCHLA/JABSC 2010;31(3): 99-107).

In this article, the author reports the results of a survey conducted by e-mail in October 2009. However, librarians from Quebec universities were excluded from the sample on the basis of two criteria.

First, the author writes that "universities with a student population similar to that of Concordia University were chosen" to participate in the survey. According to university websites, total enrolment at Concordia University for 2009-2010 was 45126 students; yet, according to Appendix $\mathrm{B}$, the University of Toronto, the institution with the largest student population in Canada (73685 in 2008-2009), was included in the survey. Thus, the size restriction was not applied thoroughly in the selection process.

Second, the author indicates that "given possible cultural differences that could impact service provision, universities in Quebec were not sampled". This statement requires further explanation, as cultural differences are certainly not considered to be a teaching or learning issue at postsecondary levels, especially in sciences. Here are examples where, as far as we know, cultural differences have no such impact:

- accreditation standards of the Committee on Accreditation of Canadian Medical Schools;
- statistics compiled by the Association of College Research Libraries; and

- the Times Higher Education's World University Rankings.

As the Health Library of Université de Montréal was not included in the survey, we would like to report that a bioinformatics service was indeed in its early stages in October 2009. Ms. Natalie Clairoux attended a Canadian Health Libraries Association-sponsored Bioinformatics Workshop (JCHLA/JABSC 2009;30(4):145-6) and interviewed a number of stakeholders to assess the interest for the library's implication in bioinformatics education. Our service began in January 2010, and includes reference, a NCBI databases workshop, and an online subject guide. Details of the implementation and evaluation of our bioinformatics service will be presented at Canadian Health Library Association's annual meeting in June 2011.

Thus, we would like the author to define more precisely how the universities were sampled for the survey. Perhaps additional criteria were used, such as the university offering or not offering a particular program, or the total student population in a particular program. Otherwise, we find it quite dismaying to exclude Quebec universities from this survey (as they all offer programs in biological or health sciences), simply on the basis of "possible cultural differences", which should at least be explained.

Sincerely,

Monique St-Jean

Directrice, Bibliothèques des sciences de la santé, Université de Montréal 